\title{
Variables asociadas al síndrome de burnout en el área de la salud de una clínica de Villavicencio, Colombia
}

\author{
Variables associated with burnout syndrome \\ in the health area of a clinic at Villavicencio, Colombia
}

\author{
Karen Tatiana Castillo Parra ${ }^{1}$, Nadyara Cestagalli Vargas ${ }^{1}$, \\ Juan Sebastián Vernaza Sánchez ${ }^{1}$, Fernando Riveros Munévar ${ }^{l}$, \\ Liliana Bernal Vargas ${ }^{2}$ y Angie Paola Quiñonez Moreno ${ }^{3}$
}

Citación: Castillo P., K.T., Cestagalli V., N., Vernaza S., J.S., Riveros M., F., Bernal V., L. y Quiñones M., A.P. (2019). Variables asociadas al síndrome de burnout en el área de la salud de una clínica de Villavicencio, Colombia. Psicología y Salud, 29(1), 51-62.

\section{RESUMEN}

\begin{abstract}
El objetivo de esta investigación descriptivo-correlacional fue establecer la relación entre las variables sociodemográficas, laborales y psicológicas asociadas al síndrome de burnout en 161 profesionales del área de la salud de una clínica de Villavicencio, Colombia, a quienes se aplicaron un cuestionario de datos sociodemográficos y laborales, los cuestionarios de Burnout de Maslach, el mos de apoyo social, el de Formas de Afrontamiento, el de Orientación hacia la Vida LOT-R y la escala de resiliencia CD-RISC. Los resultados indican que una cuarta parte de la citada muestra manifestaba un alto nivel de agotamiento y baja realización personal, una tercera parte despersonalización, y diez de cada cien el síndrome de burnout completo. Los autores discuten los resultados basándose en los estilos de afrontamiento y el apoyo afectivo percibido.
\end{abstract}

Palabras clave: Burnout; Trabajadores de la salud; Estrés laboral, Colombia.

\begin{abstract}
The objective of this descriptive-correlational study was to establish the relationship between sociodemographic, labor, and psychological variables associated with burnout syndrome in healthcare workers in a clinic at Villavicencio, Colombia. Participants were 161 professional caregivers. A socio-demographic and labor data, the Maslach, the MOS, the Coping Forms, and the LOT-R questionnaires, as well as the CD-RISC resilience scale were applied. The results show the levels of exhaustion, low personal fulfillment, depersonalization, and complete burnout syndrome in the participants. The authors discuss their results on the basis of the coping styles and perceived affective support.
\end{abstract}

Keywords: Burnout; Health workers; Work stress; Colombia.

\section{INTRODUCCIÓN}

$\mathrm{E}$ n la actualidad, las manifestaciones de estrés y las enfermedades psicosomáticas se han convertido en un fenómeno de gran interés debido a que se ha observado un aumento en su prevalencia, lo que afecta considerablemente la salud de las personas y su rendimiento laboral (Naranjo, 2009). Durante el desarrollo de las diferentes actividades laborales, las presiones y situaciones estresantes pueden originar

\footnotetext{
${ }^{1}$ Programa de Psicología, Universidad de San Buenaventura, Carrera 8H \# 172-20, 110141 Bogotá, Colombia, tel. (57)16-67-10-90, ext. 2602, correo electrónico: ktcastillop@gmail.com. Artículo recibido el 23 de marzo y aceptado el 7 de junio de 2018.

${ }^{2}$ Facultad de Ciencias Humanas y Sociales, Programa de Psicología, Universidad Cooperativa de Colombia, sede Villavicencio, Carrera 22 \# 7-06, 500001 Villavicencio, Colombia, tel. 57(8)681-88-50, ext. 8497, correo electrónico: liliana_bernal1@hotmail.com.

${ }^{3}$ Corporación Clínica, Universidad Cooperativa de Colombia, Calle 36 \# 35-62, 500001 Villavicencio, Colombia, tel. 57(8)661-43-00, ext. 305, correo electrónico:aquinonez@clinicaucc.com.
} 
efectos nocivos no solamente en el ámbito laboral, sino también en el personal, social y familiar (De la Cruz y Frutos, 2013).

El estrés es un factor que permea el vivir cotidiano, pues condiciones tales como los cambios tecnológicos, las excesivas cargas laborales y las crecientes responsabilidades producen en los trabajadores una considerable variación en sus estados emocionales, lo que genera a su vez que el nivel de estrés aumente y se sufra un deterioro global que afecta directamente su comportamiento, con lo que se manifiestan y desarrollan sintomatologías más complejas (De la Cruz y Frutos, 2013). Al respecto, el síndrome de burnout, también conocido como síndrome de desgaste profesional, y más recientemente como síndrome de quema laboral (Gil-Monte, 2007) o estrés asistencial (Rozo, 2007), se considera una enfermedad profesional caracterizada por agotamiento emocional, crisis vocacional, insatisfacción personal y fatiga, los que pueden acompañarse de muchos otros síntomas físicos, psíquicos y sociales.

El burnout, según Gil-Monte y Marucco (2008), genera un deterioro cognitivo que radica en la baja realización personal dentro de la organización debida a un deterioro afectivo caracterizado por agotamiento emocional y físico, o que se traduce en actitudes y conductas negativas hacia los clientes y hacia la propia organización.

Rozo (2007) plantea que en la dimensión cognoscitiva se aprecia más intolerancia, inflexibilidad y cierre a toda innovación; aumenta el desapego, la desconfianza, el cinismo, el pesimismo, la sospecha, la despersonalización, el aburrimiento, la omnipotencia y la hipercrítica.

Respecto a la dimensión afectiva, el mismo Rozo, apoyado en varios autores, señala que aumentan la depresión, el abatimiento, la soledad, el miedo, la fatiga y el vacío emocional, la culpabilidad, la irritabilidad, la vulnerabilidad, la tensión, el enfado y la pérdida de control, todo lo cual genera el deseo de aislarse o apartarse de las demás personas.

En cuanto a la dimensión conductual, Correal, Posada y Pérez (2000) proponen que los cambios conductuales dependen de las variables personales y ambientales; hay aburrimiento durante el trabajo, pérdida del entusiasmo, dificultad para concentrarse, baja productividad y disminución de la calidad del desempeño laboral; la persona muestra conductas agresivas; hay aumento o reducción en el consumo de comida, aumento en la accidentalidad y, en ocasiones, consumo de sustancias.

En lo tocante a la dimensión física, la revisión de Rozo (2007) muestra que las personas que sufren el síndrome de burnout pueden llegar a padecer cefaleas tensionales, dolores de columna, resfriados frecuentes, disminución del sueño, fatiga crónica, cansancio, tensión muscular, problemas gastrointestinales y, en algunos casos, cambios en el peso corporal. El mismo autor, en la dimensión de relación, indica que se ha observado que los profesionales de la salud interactúan de manera diferente con sus pacientes y colegas, tienen dificultades para comunicarse con los demás, su relación es mecánica o artificial y generan conflictos interpersonales (Rozo, 2007).

Teniendo en cuenta lo anterior, hay personas que son más vulnerables al burnout debido a ciertos factores individuales y situacionales que las hacen más propensas. Entre esos factores personales, el que sean sujetos motivados pero obsesivos, entusiastas, entregados a su trabajo y con personalidad emocional, aumenta la probabilidad de desarrollar el síndrome; así, los profesionales en las disciplinas humanitarias o asistenciales, como medicina, psicología, enfermería, trabajo social y enseñanza, muestran una elevada vulnerabilidad (Hernández, 2002). Entre los factores situacionales, son las características organizacionales de la tarea, el reconocimiento o su ausencia y los recursos de que se dispone las que desempeñan un papel fundamental en las instituciones de salud (Rozo, 2007).

Respecto a la construcción y comprensión conceptual del burnout, Maslach (2009) plantea que el foco del fenómeno radica en el proceso de erosión psicológica y en los resultados psicológicos y sociales de una exposición crónica al estrés; es decir, el burnout es el resultado de una respuesta prolongada a los estresores interpersonales crónicos en el trabajo y tiende a ser bastante estable con el tiempo.

Revisiones metaanalíticas indican que este estilo de afrontamiento desadaptativo conduce a una falta de realización personal en la que hay una pérdida de recursos debido al agotamiento; las demandas del trabajo amenazan y agotan los re- 
cursos propios; con el tiempo, la exposición prolongada a tales condiciones lleva al agotamiento emocional, el cinismo y la reducción de la realización personal (Alarcón, 2011). En otra revisión de Alarcón, Eschleman y Bowling (2009) se encontró que la personalidad del empleado está relacionada consistentemente con dicho agotamiento.

Diversos autores (Andrés, De Juan, Escobar, Jarado y Martínez, 1998; Benavides, Gimeno y Benach, 2002; Chacón y Grau, 2004) señalan algunas características o variables de tipo demográfico, como la edad, el sexo y el estado civil, y otras condiciones como el clima laboral, la profesión, el nivel de formación y la ambigüedad del rol, entre otras, como variables que cumplen una función antecedentes en el desarrollo del síndrome.

En relación al sexo, la revisión realizada por Bethelmy y Guarino (2008) apunta que ésta introduce variabilidad en los niveles de estrés laboral, en las estrategias de afrontamiento y en la sensibilidad emocional; en efecto, las mujeres suelen reportar más síntomas depresivos, un peor estado de salud general y mayores tensores crónicos y niveles de rumiación que los hombres. En esta misma línea, el estudio de Matud (2004) encontró diferencias por género en cuanto a los estilos de afrontamiento, la salud y el estrés en una muestra española de 2,816 residentes de las Islas Canarias, donde las mujeres reportaron niveles de estrés crónico, síntomas somáticos y estrés psicológico significativamente mayores que los hombres. En cuanto a los estilos de afrontamiento y el estilo emocional adoptado, los hombres utilizaron las estrategias de afrontamiento racional y la de desapego e inhibición emocional en mayor medida que las mujeres, y éstas mostraron un mayor uso de las estrategias de afrontamiento emocional y evitación que aquéllos.

Respecto al sexo, los estudios de Bergman, Ahmad y Stewart (2003) hallaron diferencias en el servicio médico en cuanto al estrés laboral, la salud física y mental, la satisfacción laboral, el estado civil, los estilos de vida saludable, las cargas laborales y el apoyo social cuando se experimenta estrés. En un análisis de regresión simple se encontró que, en las mujeres, la varianza de síntomas somáticos se explica por el apoyo de los pares en situaciones de estrés y por la carga laboral, mientras en los hombres lo hace por la salud mental, el apoyo social cuando se está estresado y la carga laboral. Independientemente del sexo, los médicos muestran altas demandas o cargas laborales.

Olmedo (1997) y Chacón y Grau (2004) afirman que la autoestima y la autoeficacia afectan la manera en que los individuos desarrollan actitudes respecto a sí mismos, la que incide en su desempeño laboral y en el afrontamiento que realizan ante las condiciones estresantes de su puesto de trabajo. En relación al burnout y la resiliencia, tres estudios (Gil y Peiró, 1997; Moreno, González y Garrosa, 2002; Ríos, Carrillo y Sabuco, 2012) indican que la resiliencia desempeña un importante papel protector en el surgimiento del cuadro de desgaste profesional o síndrome de burnout. Sobre ello, se han relacionado los aspectos positivos de la resiliencia con el engagement (compromiso) y la menor vulnerabilidad de padecer dicho síndrome (Menezes, Fernández, Hernández, Ramos y Contador, 2006), concluyéndose que los cuidadores más resilientes no llegan a experimentarlo, sino que logran más habilidades y competencias de compromiso laboral, y que en la medida que refuerzan estos atributos, mitigan su vulnerabilidad al burnout.

Kaschka, Korczak y Broich (2011) evidencian que, en Alemania, el índice de prevalencia de este problema ha tenido un rápido incremento, mientras que el Instituto de Investigaciones sobre Política, Medios de Comunicación y de Opinión de Alemania (TNS Enmid) (cf. Kaschka et al., 2011) encontró que $12.5 \%$ de todos los empleados de ese país se sentían abrumados en sus puestos de trabajo. Taris, Peeters, Le Blanc, Schreurs y Schaufeli (2001) llevaron a cabo un estudio con docentes, hallando que la inequidad en la relación entre un alto esfuerzo y una baja recompensa, particularmente la establecida con los receptores de sus servicios profesionales (esto es, los estudiantes), se asoció con estresores provenientes de estos últimos, así como con los componentes del síndrome de burnout. De tal suerte, este enfoque confirma, en el caso del burnout, la hipótesis que subyace al modelo de estrés, o sea, el desbalance entre esfuerzo y recompensa propuesto por Siegrist (1996), aplicable no solo al estrés en las relaciones sociales, sino en general a la relación del hombre con el trabajo. 
Investigaciones realizadas en países como Estados Unidos y algunos europeos, como lo indican Moreno, González y Garrosa (2001), De las Cuevas (2003), Mingote, Moreno y Gálvez (2004) y Chacón y Grau (2004), sugieren la necesidad de establecer los niveles de salud y estrés en los profesionales de la medicina debido a que se les considera actualmente como una población vulnerable de padecer enfermedades físicas y psicológicas originadas por el estrés laboral en el ejercicio de su profesión (Gjerløw, 2001).

En un estudio desarrollado por García y Herrero (2008) se encontró una prevalencia mayor de síntomas de despersonalización en hombres que en mujeres, siendo así la única dimensión con diferencias significativas de acuerdo al género; por el contrario, variables como la edad, el tiempo de ejercicio en la profesión y el número de hijos no mostraron diferencias considerables. En cuanto a diferencias según la profesión, los datos normativos obtenidos de una población norteamericana identifican a los profesionales de la salud como uno de los grupos con medias más altas en los diferentes componentes del síndrome. Esto conlleva la necesidad de clarificar el proceso del burnout, específicamente entre profesionales del área de la salud.

De acuerdo a los criterios diagnósticos del síndrome, en un estudio publicado por Gil-Monte y Marucco (2008), quienes evaluaron a 123 pediatras con los criterios de detección de burnout en países como España, Argentina, Holanda y Estados Unidos, los resultados indican que, según los puntos de corte estipulados para Estados Unidos, la prevalencia fue de $10.6 \%$, aumentando a $24.4 \%$ al ajustarse a los criterios de España. Este aumento fue aún mayor con los criterios empleados en Argentina (37.4\%); en cambio, considerando los criterios clínicos establecidos en Holanda, el porcentaje se redujo a $3.2 \%$, lo que demuestra que hay una dificultad general para determinar aspectos tales como la prevalencia del síndrome, que es un producto de la variabilidad de los criterios en las puntuaciones de corte.

Pérez, Iruarrizaga y Camuñas (2001) identificaron una alta relación entre la ansiedad generalizada a situaciones de la vida cotidiana y el desarrollo de burnout en personal hospitalario. Mostraron que la ansiedad fisiológica se asociaba más al cansancio emocional, en tanto que la ansiedad cognitiva se hallaba más relacionada con la despersonalización. En un estudio realizado por Grau, Suñar y García (2005) con 2,290 empleados de cinco hospitales sanitarios de Girona, se hallaron asociaciones inversas entre la despersonalización y variables tales como los años dedicados al ejercicio de la profesión, el optimismo, la valoración de ser útiles en el trabajo y la sensación de ser valorado por otros, en tanto que altos niveles de cansancio emocional se asociaron inversamente con el optimismo, la valoración de la utilidad en el trabajo, el equipo de trabajo y la realización personal.

Ahora bien, los hallazgos son en ocasiones contradictorios entre sí, tal como ocurre con los de Díaz, Castro, Mendo y Olcese (2006), quienes revisaron los factores demográficos y el síndrome de burnout en practicantes de enfermería y psicología, teniendo en cuenta la relación entre algunos aspectos demográficos, como edad, sexo y estado civil, respecto al síndrome. En dicho estudio se obtuvo como resultado que los dos grupos participantes manifestaban un bajo agotamiento emocional y expresaban sentirse satisfechos personal y profesionalmente, mientras que el estudio de Sabogal, Herrera y Farfán (2015), hecho con trabajadores del área de la salud, como médicos, enfermeras, obstetras y nutricionistas de entre 18 y 65 años de edad, mostró que no había relación alguna entre el estilo de vida y el síndrome de burnout, y tampoco entre el agotamiento emocional y el estilo de vida con la despersonalización; solo se encontró una relación significativa entre el estilo de vida y la dimensión de realización personal.

En lo que respecta al burnout en trabajadores colombianos de la salud, Hernández (2002) realizó un estudio en el cual evaluó al personal sanitario de un hospital castrense, detectando manifestaciones de agotamiento emocional y baja realización personal como expresiones principales del síndrome, pero no síntomas de despersonalización. A su vez, Contreras, Juárez y Murrain (2008) valoraron a un grupo de auxiliares y profesionales de enfermería de Bogotá con el propósito de identificar las estrategias de afrontamiento ante el burnout, así como la calidad de vida y los factores socioeconómicos. En dicho estudio, identificaron una prevalencia baja del síndrome de burnout (18.3\% 
con severidad baja, $1.4 \%$ moderada y $2.8 \%$ alta), en contraste con los datos referenciales empleados, así como que entre más ajustados estaban los individuos a las condiciones sociodemográficas susceptibles de generar burnout, mejores eran sus respuestas de afrontamiento.

Un análisis de Muñoz, Osorio, Robles y Romero (2014), hecho en el hospital de Ibagué con enfermeras, trató de determinar la relevancia del síndrome en dicho personal e identificar su relación con las variables sociodemográficas. El estudio se realizó en una muestra aleatoria y representativa de 174 enfermeras, clasificadas como profesionales o auxiliares. Los resultados mostraron que la prevalencia de burnout fue de $20.1 \% \mathrm{y}$ que $52.3 \%$ de las participantes en las pruebas se hallaban en riesgo de padecerlo. Quienes manifestaron no tener una pareja tenían una vinculación temporal con el síndrome; las personas con compromisos sentimentales y un contrato laboral fijo fueron quienes lo sufrían en mayor proporción, al igual que los hombres o mujeres con hijos.

Adicionalmente, en una revisión metaanalítica llevada a cabo por Gómez (2017) se halló que la prevalencia del síndrome de burnout en enfermeras de urgencias era elevada, y que aproximadamente un tercio de la muestra manifestaba afectaciones en al menos una de las tres subescalas de la escala de Maslach. El autor concluye que las condiciones de trabajo y los factores personales deben tenerse en cuenta al evaluar los perfiles de riesgo de agotamiento en las enfermeras de urgencias.

Por otra parte, Rodríguez, Rodríguez, Riveros, Rodríguez y Pinzón (2010) realizaron un estudio del personal de la salud en tres instituciones de Bogotá, en el cual se tuvieron en cuenta profesiones específicas. Sus resultados indican que la prevalencia del burnout fue de $20.6 \%$, y que $30.4 \%$ de los profesionales se encontraban emocionalmente agotados en el trabajo algunas veces al mes, aunque la cuarta parte informó que ello les ocurría varias veces a la semana. De los participantes, $19 \%$ se sentían cansados una vez o menos al mes cuando se levantaban por las mañanas e iniciaban otro día de trabajo, y $4.9 \%$ dijeron experimentar esa sensación diariamente.

Por todo lo anterior, el burnout constituye un problema creciente que resulta relevante para la psicología dado que la evidencia empírica de- muestra que no sólo entraña una problemática social, sino también una de salud pública porque requiere una atención inmediata. Si bien la literatura en general es amplia acerca del burnout, las investigaciones realizadas en Colombia aún son limitadas.

Por lo anterior, la presente investigación cuantitativa, transversal y de alcance descriptivo-correlacional (Hernández, Fernández y Baptista, 2014) tuvo como propósito determinar la relación entre variables sociodemográficas, laborales y psicológicas asociadas al síndrome de burnout en trabajadores del área de la salud de una clínica ubicada en la ciudad de Villavicencio (Colombia).

\section{MÉTODO}

\section{Participantes}

Participaron 161 trabajadores del área médicoasistencial, de 18 a 64 años de edad $(\mathrm{M}=30.81$; D.E. $=8.0$ ), obtenidos por medio de un muestreo no probabilístico por sujetos disponibles. En la Tabla 1 se muestran los datos sociodemográficos generales de los mismos.

Tabla 1. Datos sociodemográficos de la muestra.

\begin{tabular}{|l|r|r|}
\hline Estado civil & n & \% \\
\hline Solteros & 68 & 42.2 \\
\hline Casados & 39 & 24.2 \\
\hline Unión libre & 41 & 25.5 \\
\hline Separados/divorciados & 2 & 1.2 \\
\hline Viudos & 1 & 0.6 \\
\hline Estrato & 21 & 13.0 \\
\hline Estrato 1 & 45 & 28.0 \\
\hline Estrato 2 & 57 & 35.4 \\
\hline Estrato 3 & 13 & 8.1 \\
\hline Estrato 4 & 2 & 1.2 \\
\hline Estrato 5 & 2 & 1.2 \\
\hline Estrato 6 & 13 & 11.2 \\
\hline Sexo & 23 & 83.2 \\
\hline Femenino & 134 \\
\hline Masculino & \\
\hline Diagnóstico actual & & 85.7 \\
\hline Sí & 138 \\
\hline No & \multicolumn{2}{|l|}{} \\
\hline
\end{tabular}


En la Tabla 2 se presentan los datos laborales de los participantes.

Tabla 2. Datos laborales de la muestra.

\begin{tabular}{|c|c|c|}
\hline Área general de trabajo & n & $\%$ \\
\hline Hospitalización & 73 & 73.0 \\
\hline $\begin{array}{l}\text { Unidad de cuidados } \\
\text { intensivos neonatales }\end{array}$ & 15 & 15.0 \\
\hline Cirugía & 24 & 24.0 \\
\hline UCI intermedio & 30 & 30.0 \\
\hline Urgencias & 8 & 8.0 \\
\hline \multicolumn{3}{|l|}{ Horas de trabajo } \\
\hline $5-9$ & 59 & 36.6 \\
\hline $10-13$ & 65 & 40.3 \\
\hline $14-18$ & 23 & 14.3 \\
\hline \multicolumn{3}{|l|}{ Nivel de formación } \\
\hline Técnico & 80 & 49.7 \\
\hline Universitario & 59 & 36.6 \\
\hline Especialista & 20 & 12.4 \\
\hline Magister & 2 & 1.2 \\
\hline \multicolumn{3}{|l|}{ Profesión } \\
\hline Médico & 24 & 14.9 \\
\hline Psicólogo & 1 & 0.6 \\
\hline Enfermero & 82 & 50.9 \\
\hline Enfermero jefe & 22 & 13.7 \\
\hline Otra & 29 & 18.0 \\
\hline \multicolumn{3}{|l|}{ Remuneración } \\
\hline $\mathrm{Si}$ & 24 & 14.9 \\
\hline No & 121 & 75.2 \\
\hline \multicolumn{3}{|c|}{ Salario (en pesos colombianos) ${ }^{4}$} \\
\hline $500.000-3.000 .000$ & 122 & 75.3 \\
\hline $3.000 .050-6.000 .000$ & 10 & 6.1 \\
\hline $6.000 .050-9.000 .000$ & 2 & 1.2 \\
\hline $9.000 .050-12.000 .000$ & 3 & 1.8 \\
\hline \multicolumn{3}{|l|}{ Tiempo en el cargo } \\
\hline $1-6$ & 103 & 68.6 \\
\hline $7-12$ & 15 & 10.0 \\
\hline $13-18$ & 8 & 5.5 \\
\hline $19-25$ & 4 & 2.6 \\
\hline \multicolumn{3}{|l|}{ Tiempo en la profesión } \\
\hline $1-7$ & 57 & 38.1 \\
\hline $8-14$ & 22 & 14.0 \\
\hline $15-21$ & 10 & 6.7 \\
\hline $22-28$ & 3 & 2.1 \\
\hline
\end{tabular}

${ }^{4}$ Donde 10,000 colombianos equivalen a 3.41 dólares.

\section{Instrumentos}

Se utilizó un cuestionario de trece preguntas sobre variables sociodemográficas y laborales, elaborado ex profeso para obtener los datos personales de cada participante, así como información sobre sus condiciones de trabajo.

Inventario de Burnout de Maslach para el Desgaste Profesional (MBI-HSS) (Maslach y Jackson, 1986).

Es una escala que contiene 22 ítems que se evalúan en una escala tipo Likert con cinco opciones de respuestas. Cuenta con tres subescalas: agotamiento emocional, despersonalización y realización personal. Se dispuso de la versión adaptada del instrumento para Cali, Colombia (Córdoba et al., 2011), que mantiene los componentes originales. Posee una consistencia interna de Cronbach de .77.

Cuestionario de Estilos de Afrontamiento (CEA) (Folkman y Lazarus, 1988).

Para medir el afrontamiento, este cuestionario, revisado por Vitaliano, Russo, Carr, Maiuro y Becker (1985) y traducido al español y adaptado por Flórez (1993), es un instrumento que consta de 42 ítems, con opciones de respuesta tipo Likert en un rango que va de 1 a 4, desde "Para nada" hasta "En gran medida". Para esta investigación se utilizó la versión adaptada para Colombia por Rueda y Vélez (2010), que conserva los componentes originales. Tiene una confiabilidad obtenida por el coeficiente alfa de Cronbach de .87 .

\section{Medical Outcomes Study - Social Support Survey} (MOS) (Stewart y Ware, 1992).

Para la medición de apoyo social se utilizó este cuestionario, que se compone de 23 ítems con cinco opciones de respuesta tipo Likert. Evalúa un índice global de apoyo social, apoyo emocional, apoyo afectivo, relaciones sociales de ocio y distracción $\mathrm{y}$ ayuda material. Validado para población normal en Colombia por Londoño et al. (2012), cuenta con un índice de confiabilidad de .941.

Connor-Davidson Resilience Scale (CD-RISC-10) (Campbell y Stein, 2007).

En cuanto a la resiliencia, se empleó esta escala de diez ítems en formato tipo Likert con cinco opciones de respuesta, que van de 0 a 4 puntos. Para 
este estudio se utilizó la versión adaptada al español por Notario et al. (2014), misma que cuenta con los datos de validez de Riveros, Bernal, Bohórquez, Vinaccia y Quiceno (2016), en versión que mantuvo los componentes originales de la escala y que tiene una confiabilidad de .82 .

Life Orientation Test (versión revisada) (LOT-R) (Scheier y Carver, 1985).

Para medir optimismo se empleó este instrumento de diez ítems, misma que se califica en una escala tipo Likert de cinco puntos, con tres elementos para el optimismo, tres para el pesimismo y cuatro elementos de relleno, que se incluyen para cubrir el objetivo del instrumento. Las puntuaciones de las subescalas de optimismo y pesimismo se calculan sumando los puntos obtenidos en los elementos correspondientes. La puntuación total de la suma se calcula sumando los puntajes brutos de la subescala de optimismo con los puntajes brutos invertidos de la subescala de pesimismo. Para este estudio se utilizó la validación y los datos normativos para Colombia obtenidos por Zenger et al. (2013), en versión que mantiene los componentes originales de la escala y que cuenta con un coeficiente alfa de Cronbach de .58.

\section{Procedimiento}

Después de establecer un convenio con la clínica de Villavicencio para que permitiera el acceso a la población, se hizo entrega de una carta de consentimiento informado para que cada participante estuviese al tanto de los propósitos de la investigación, tras de lo cual se aplicaron los cuestionarios e instrumentos previamente indicados. Cabe referir que las aplicaciones se realizaron en un salón, en silencio y sin interrupciones, a fin de garantizar el control de variables extrañas.

\section{Análisis de datos}

Por medio del paquete estadístico SPSS, versión 24 , se procedió a hacer un análisis descriptivo y uno de normalidad; luego, según dicho criterio de normalidad, se determinaron los coeficientes de correlación y, con base en ellos, se procedió a llevar a cabo un análisis de regresión lineal múltiple.

\section{RESULTADOS}

Con el fin de mostrar los hallazgos y la caracterización de la muestra a la luz de los objetivos de la investigación, se incluye en orden lo encontrado respecto a la MBI-HSS, el análisis de las correlaciones y comparaciones, y finalmente los análisis de regresión. En lo correspondiente a las subescalas de burnout, se muestran en la Tabla 3 los criterios de calificación según los percentiles para cada subcomponente.

Tabla 3. Criterios de calificación de los componentes del burnout.

\begin{tabular}{|c|c|c|c|}
\hline Percentiles & Agotamiento & Despersonalización & $\begin{array}{c}\text { Realización } \\
\text { personal }\end{array}$ \\
\hline 25 & 14.00 & 5.00 & 28.00 \\
\hline 50 & 17.00 & 7.00 & 33.00 \\
\hline 75 & 23.00 & 9.00 & 38.00 \\
\hline
\end{tabular}

Nota: Abajo del percentil 25 indica un bajo nivel de la variable, mientras que arriba del percentil 75 , un alto nivel.

Teniendo en cuenta los criterios de la Tabla 3 y las frecuencias mostradas, se halló que $26.5 \%$ de los participantes manifestó un nivel alto de agotamiento emocional, $31.7 \%$ una alta despersonalización y $26.5 \%$ una baja realización personal. Con los resultados presentados anteriormente, $\mathrm{y}$ entendiendo que las personas con alto agotamiento, alta despersonalización y baja realización per- sonal configuran el síndrome en su totalidad, se puede afirmar que $10 \%$ de la muestra lo padecía.

Con el fin de determinar si las variables presentaban una distribución normal, se llevó a cabo la prueba de Kolmogorov-Smirnov, encontrando que ninguna de las variables obtuvo significancias superiores a 0.05 , por lo cual se hicieron análisis no paramétricos, y para determinar las diferen- 
cias entre las variables según el sexo se utilizó la prueba U de Mann-Whitney, hallando que la despersonalización alcanzó niveles más altos en hombres $(\mathrm{U}=1246.500$; sig. $=0.047)$ y que la búsqueda de soporte social fue mayor en mujeres $(\mathrm{U}=677.500$; sig. $=0.016)$.

Por otro lado, al establecer la relación entre los componentes del burnout y las variables labo- rales y psicológicas, no se obtuvieron relaciones estadísticamente significativas con las laborales (tiempo como profesional, tiempo en el cargo, horas de trabajo al día y salario). Respecto a las variables psicológicas, los resultados se muestran en la Tabla 4, teniendo en cuenta que se utilizaron coeficientes de correlación de Spearman, donde una correlación es significativa si es superior a 0.3.

Tabla 4. Resultados de las correlaciones entre los componentes del burnout y las variables psicológicas.

\begin{tabular}{|c|c|c|c|c|}
\hline & & Agotamiento & Despersonalización & $\begin{array}{c}\text { Realización } \\
\text { personal }\end{array}$ \\
\hline \multirow{2}{*}{$\begin{array}{l}\text { Afrontamiento } \\
\text { evitativo }\end{array}$} & Coeficiente de correlación & $.309 * *$ & 0.090 & -0.137 \\
\hline & Sig. (bilateral) & 0.001 & 0.325 & 0.132 \\
\hline \multirow{2}{*}{$\begin{array}{l}\text { Apoyo } \\
\text { emocional }\end{array}$} & Coeficiente de correlación & $-.322 * *$ & $-.198 *$ & $.350 * *$ \\
\hline & Sig. (bilateral) & 0.000 & 0.016 & 0.000 \\
\hline \multirow{2}{*}{$\begin{array}{l}\text { Relaciones sociales } \\
\text { de ocio y distracción }\end{array}$} & Coeficiente de correlación & $-.265 * *$ & -0.112 & $.347 * *$ \\
\hline & Sig. (bilateral) & 0.001 & 0.176 & 0.000 \\
\hline \multirow{2}{*}{ Apoyo afectivo } & Coeficiente de correlación & $-.303 * *$ & $-.195 *$ & $.338 * *$ \\
\hline & Sig. (bilateral) & 0.000 & 0.018 & 0.000 \\
\hline \multirow{2}{*}{ Optimismo } & Coeficiente de correlación & $-.242 * *$ & $-.317 * *$ & $.266 * *$ \\
\hline & Sig. (bilateral) & 0.004 & 0.000 & 0.001 \\
\hline
\end{tabular}

**Correlaciones estadísticamente significativas $(p=0.05)$.

Tales resultados indican que el agotamiento mostró una correlación positiva y significativa con el afrontamiento evitativo, y correlaciones negativas con el apoyo emocional y el apoyo afectivo. Por su parte, la despersonalización correlacionó negativamente con los niveles de optimismo, y finalmente la realización personal arrojó correlaciones directas con el apoyo emocional, relaciones sociales de ocio y distracción y apoyo afectivo. También se obtuvieron las correlaciones entre los componentes del burnout y el soporte social, siendo segmentadas por el sexo de los participantes, donde se halló en los hombres una correlación positiva entre el agotamiento y la búsqueda de soporte social.

\section{Variables predictoras de los componentes del burnout}

Con el fin de determinar cuáles eran las variables que predicen los componentes del burnout (agotamiento, despersonalización y realización personal), y teniendo en cuenta el tipo de variables de orden numérico en las variable de criterio y en las predictoras, se llevaron a cabo análisis de regresión lineal múltiple. Cabe indicar que se garantizaron los supuestos de colinealidad y homocedasticidad en las varianzas, este último requisito mediante la prueba de Levene. Los resultados se muestran para cada componente iniciando con el agotamiento (Tabla 5).

Tabla 5. Modelo de regresión para el agotamiento.

\begin{tabular}{|c|c|c|c|c|}
\hline Modelo & $\mathbf{R}$ & $\mathbf{R}^{2}$ & $\begin{array}{c}\mathbf{R}^{2} \\
\text { ajustado }\end{array}$ & $\begin{array}{c}\text { Cambio } \\
\text { en }^{2}\end{array}$ \\
\hline $\mathbf{1}$ & $.507^{\mathrm{a}}$ & .257 & .243 & .257 \\
\hline $\mathbf{2}$ & $.592^{\mathrm{b}}$ & .350 & .324 & .093 \\
\hline $\mathbf{3}$ & $.636^{\mathrm{c}}$ & .404 & .368 & .054 \\
\hline
\end{tabular}

\footnotetext{
a. Predictores: (Constante), Apoyo afectivo.

b. Predictores: (Constante), Apoyo afectivo, Afrontamiento evitativo.

c. Predictores: (Constante), Apoyo afectivo, Afrontamiento evitativo, Búsqueda soporte social.

d. Variable dependiente: Agotamiento.
}

La tabla anterior permite entonces indicar tres modelos, siendo el tercero el de mejor ajuste, pues 
muestra que el apoyo afectivo, los estilos de afrontamiento evitativo y la búsqueda de soporte social logran predecir $36.8 \%$ de la variación en el agotamiento. Los coeficientes estandarizados beta hallados muestran que entre menor es el apoyo afectivo (-.334), mayor el uso que se hace del estilo de afrontamiento evitativo (.339) y menor la búsqueda de soporte social (-.262), mayores niveles de agotamiento se pueden predecir.

Sobre el componente de despersonalización, se hicieron análisis separados de regresión por cada sexo debido a la diferencia en esta variable entre ellos. Los resultados se muestran en la Tabla 6.

Tabla 6. Modelos de regresión para la despersonalización, segmentados por sexo

\begin{tabular}{l|c|c|c|c|c|}
\hline \multicolumn{1}{|c|}{ Sexo } & Modelo & $\mathbf{R}$ & $\mathbf{R}^{2}$ & $\begin{array}{c}\mathbf{R}^{\mathbf{2}} \\
\text { ajustado }\end{array}$ & $\begin{array}{c}\text { Cambio } \\
\text { en } \mathbf{R}^{\mathbf{2}}\end{array}$ \\
\hline Hombre & 1 & $.673 \mathrm{a}$ & .453 & .399 & .453 \\
\hline Mujer & 1 & $.559 \mathrm{c}$ & .313 & .295 & .313 \\
\hline
\end{tabular}
a. Predictores: (Constante), Afrontamiento dirigido al problema.
c. Predictores: (Constante), Apoyo afectivo.

Dicha tabla refiere un único modelo para cada sexo, que explica para los hombres $39.9 \%$ y para las mujeres $29.5 \%$ de la varianza. Entre los primeros, un menor uso del afrontamiento dirigido al problema $(\beta=-.673)$, y entre las segundas el menor apoyo afectivo percibido $(\beta=-.559)$ predicen mayores niveles de despersonalización. Finalmente, en lo referente a la realización personal (Tabla 7), se obtuvieron dos modelos, el segundo con un mejor ajuste, que explica $38.3 \%$ de la varianza, hallándose que un mayor uso del afrontamiento dirigido al problema $(\beta=.511)$ y un mayor apoyo material percibido $(\beta=.258)$ predicen de mejor manera la realización personal.

Tabla 7. Modelos de regresión para la realización personal.

\begin{tabular}{|c|c|c|c|c|}
\hline Modelo & $\mathbf{R}$ & $\mathbf{R}^{\mathbf{2}}$ & $\begin{array}{c}\mathbf{R}^{\mathbf{2}} \\
\text { ajustado }\end{array}$ & $\begin{array}{c}\text { Cambio } \\
\text { en }^{\mathbf{2}}\end{array}$ \\
\hline 1 & $.588 \mathrm{a}$ & .346 & .333 & .346 \\
\hline 2 & $.638 \mathrm{~b}$ & .407 & .383 & .061 \\
\hline
\end{tabular}

a. Predictores: (Constante), Afrontamiento dirigido al problema.

b. Predictores: (Constante), Afrontamiento dirigido al problema, Ayuda material.

\section{DISCUSIÓN}

La presente investigación tuvo como objetivo determinar la relación entre variables sociodemográficas, laborales y psicológicas asociadas al síndrome de burnout en el área de la salud en una clínica de la ciudad de Villavicencio (Colombia), contando con 161 participantes distribuidos en las distintas áreas de la clínica, como hospitalización, cirugía, unidad de cuidados intensivos neonatales, unidad de cuidados intensivos intermedios y urgencias.

Richardsen y Burke (1991) encontraron que las profesiones de la salud tienen altas demandas del trabajo que generan el síndrome de burnout, como se puede apreciar en los resultados de este estudio.

De acuerdo con los resultados, $26.5 \%$ de la muestra manifestaba un alto nivel de agotamiento, $27.5 \%$ una baja realización personal y $31.7 \%$ despersonalización, concluyéndose que $10 \%$ de los participantes sufrían dicho síndrome, teniendo en cuenta que para configurarlo deben reunirse los tres elementos citados.

En comparación con los resultados obtenidos, Kaschka et al. (2011) planteó que la prevalencia del síndrome en Alemania fue de 12.5\%, en tanto que Gil-Monte y Marucco (2008), según los puntos de corte estipulados para Estados Unidos, hallaron una prevalencia de $10.6 \%$, cifra que aumenta a $24.4 \%$ al ajustarse a los criterios de España; en Argentina, la cifra asciende a 37.4\%, mientras que en Holanda se reduce a 3.2\%. Lo anterior indica una considerable dificultad general, producto de la variabilidad en los criterios en las puntuaciones de corte, para determinar aspectos tales como la prevalencia del síndrome. Los datos obtenidos por Contreras et al. (2008) dan cuenta de una prevalencia baja del síndrome en Bogotá (severidad baja, 18.3\%, moderada, 1.4\% y alta, 2.8\%). Rodríguez et al. (2010) señalan que la prevalencia del síndrome fue de $20.6 \%$, mientras que Muñoz et al. (2014) apuntan una prevalencia de $20.1 \%$, y que un $52.3 \%$ adicional de las personas participantes estaban en riesgo de padecer el síndrome. Lo anterior muestra que la prevalencia del síndrome de burnout oscila entre 10 y $20 \%$, coincidiendo con lo hallado en la presente investigación, y señalando además que un gran porcentaje muestral está propenso a desarrollarlo. 
Estudios como el de Hernández (2002), quien detectó manifestaciones de agotamiento emocional en Colombia, y de Rodríguez et al. (2010), quienes encontraron que $30.4 \%$ de los profesionales se encontraban emocionalmente agotados en el trabajo algunas veces al mes, $25.5 \%$ varias veces a la semana, $19 \%$ una vez al mes o menos y $4.9 \%$ diariamente, también refieren hallazgos similares a los del presente estudio. En cuanto a las correlaciones, García y Herrero (2008), al emplear variables como la edad y el tiempo de ejercicio en la profesión, no revelaron diferencias considerables, al igual que lo ocurrido en el presente trabajo.

En cuanto al sexo, Matud (2004) halló diferencias en los estilos de afrontamiento, dando cuenta de que los hombres utilizaron en mayor medida la estrategia de afrontamiento racional, y las mujeres mayores niveles de afrontamiento evitativo. El estudio de Bergman et al. (2003) mostró que los componentes del burnout en ambos sexos estuvieron altamente relacionados con las habilidades de afrontamiento y el apoyo social, lo cual contrasta con esta investigación en virtud de que aquí se encontró que la búsqueda de soporte social era mayor en las mujeres, así como una correlación directa con el afrontamiento evitativo, el apoyo emocional y las relaciones de ocio y distracción, y una correlación negativa con el apoyo emocional y afectivo. Se obtuvieron correlaciones entre los componentes del burnout y el soporte social, pues los hombres mostraron una correlación directa entre el agotamiento y la búsqueda de soporte social. Al igual que lo hallado por Bergman et al. (2003) y Matud (2004), lo anterior implica que el burnout se correlaciona con distintos estilos de afrontamiento y apoyo social según el sexo.

Por otra parte, en lo que respecta al análisis de regresión, se halló en general que los estilos de afrontamiento y el apoyo social fueron los mejores predictores de las respuestas asociadas al burnout. Este resultado es similar al de Bergman et al. (2003), quienes encontraron que los síntomas somáticos en las mujeres se explican por el apoyo de los pares en situaciones de estrés y por la carga laboral, mientras que en los hombres dichos síntomas lo hacen por el apoyo social cuando se está estresado y por las altas demandas o cargas laborales.
Es importante tener en cuenta que la predicción de variables genera un insumo teórico con el cual trabajar para disminuir los niveles de burnout y mitigar sus consecuencias, para lo cual es necesario potenciar los estilos de afrontamiento y el apoyo social; son esas variables las que deberían trabajarse en programa de intervención futuros.

En cuanto a las limitaciones de la investigación, se resalta la dificultad para acceder a la población y a la institución donde se hizo el estudio; de hecho, se requirió un extenso proceso de documentación y validación de la información para lograr que la institución aceptara participar, lo que, sumado al desinterés mostrado por algunos miembros del personal asistencial, dificultó reunir una muestra mayor. Otro aspecto a resaltar es el volumen del paquete de instrumentos, lo que pudo afectar los periodos de atención de los participantes y aumentar el sesgo de las respuestas.

Debe referirse también como limitante el que, debido a la no normalidad, el nivel de generalización disminuye y la posibilidad de falsos positivos aumenta, por lo que se requiere replicar los procedimientos para reducir tales problemas metodológicos.

Este es un estudio que se llevó a cabo en una sola institución. En futuras investigaciones se recomienda manejar las mismas variables asociadas al burnout en otras instituciones hospitalarias y emplear otros tamaños muestrales, de tal manera que se puedan hacer comparaciones estadísticas que permitan una mayor generalización de los resultados.

Para finalizar, el presente estudio tuvo como principal resultado el que el burnout en el área de la salud puede predecirse mediante los estilos de afrontamiento utilizados y la percepción de apoyo social, por lo que se espera que se generen programas de intervención basados en esta evidencia que se encaminen a favorecer el uso de estilos de afrontamiento apropiados y mejorar la sensación de apoyo social por parte de los trabajadores, lo que implicaría disminuir los niveles de agotamiento y despersonalización y aumentar la sensación de autorrealización, mejorando con ello la calidad de vida de los trabajadores en el área de la salud. 


\section{REFERENCIAS}

Alarcón, G. (2011). A meta-analysis of burnout with job demands, resources, and attitudes. Journal of Vocational Behavior, 79, 549-562. doi: 10.1016/j.jvb.2011.03.007.

Alarcón, G., Eschleman, K. y Bowling, N. (2009). Relationships between personality variables and burnout: A meta-analysis. International Journal of Work, Health and Organization, 23(3), 244-263.

Andrés, P., De Juan, D., Escobar, J., Jarabo J. y Martínez, M. (1998). Burnout: técnicas de afrontamiento de la investigación. Jornades de Foment de la Investigación. Castellón de la Plana (España): Universidad Jaume I.

Benavides I., G., Gimeno, D. y Benach, C. (2002). Descripción de los factores de riesgo psicosocial en cuatro empresas. Gaceta Sanitaria, 16(3), 222-229.

Bergman, B., Ahmad, F. y Stewart, D.E. (2003). Physician health, stress and gender at a University Hospital. Journal of Pychosomatic Research, 54, 171-178.

Bethelmy, L. y Guarino, L. (2008). Estrés laboral, afrontamiento, sensibilidad emocional y síntomas físicos y psicológicos en médicos venezolanos. Revista Colombiana de Psicología, 17, 43-58.

Campbell, L. y Stein, M. (2007). Psychometric analysis and refinement of the Connor-Davidson resilience scale (CD-RISC): validation of a 10-item measure of resilience. Journal of Trauma and Stress, 20, 1019-1028.

Chacón, M. y Grau, J. (2004). Burnout y variables personales moduladoras en enfermeros que trabajan en hospitales oncológicos. Psicología y Salud, 14(1), 67-78.

Contreras, F., Juárez, F. y Murrain, E. (2008). Influencia del burnout, la calidad de vida y los factores socioeconómicos en las estrategias de afrontamiento utilizadas por los profesionales y auxiliares de enfermería. Pensamiento Psicológico, 4(11), 29-44.

Córdoba, L., Tamayo, J., González, M., Martínez, M., Rosales, A. y Barbato, S. (2011). Adaptation and validation of the Maslach Burnout Inventory-Human Services Survey in Cali, Colombia. Colombia Médica, 4(3), 286-293.

Correal, M., Posada, M. y Pérez, L. (2000). El sindrome de burnout: conceptualización, evaluación y prevención. Tesis inédita de pregrado en Psicología. Bogotá: Universidad Católica de Colombia.

De la Cruz, E. y Frutos, M. (2013). Estrés laboral y síndrome de burnout en los profesionales de enfermería de atención hospitalaria. Tesis de pregrado en Enfermería. Valladolid (España): Universidad de Valladolid.

De las Cuevas, C. (2003). Burnout en los profesionales de la atención a las drogodependencias. Trastornos Adictivos, 5, 107-113.

Díaz, N., Castro, M., Mendo, T. y Olcese, C. (2006). Factores demográficos y síndrome de burnout en practicantes de enfermería y psicología. Revista de Psicología, 9(1), 29-39.

Flórez, L. (1993). La evaluación del estilo de afrontamiento. Boletín de la Asociación Colombiana de Psicología de la Salud, 2(1), 7-9.

Folkman, S. y Lazarus, R.S. (1988). Manual for the Ways of Coping Questionnaire Research Edition. Palo Alto, CA: Consulting Psychologists Press.

García, J.M. y Herrero, S. (2008). Variables sociodemográficas y síndrome de burnout en una muestra multiocupacional del Hospital Psiquiátrico Penitenciario de Sevilla. Apuntes de Psicología, 26(3), 459-477.

Gil-Monte, P. (2007). El sindrome de quemarse por el trabajo (burnout): una perspectiva histórica. Madrid: Pirámide.

Gil-Monte, P. y Marucco, M. (2008). Prevalencia del síndrome de quemarse por el trabajo (burnout) en pediatras de hospitales generales. Revista de Saúde Pública, 42(3), 450-456.

Gil-Monte, P.R. y Peiró, J.M. (1997). Desgaste psíquico en el trabajo: el síndrome de quemarse. Madrid: Síntesis.

Gjerløw, O. (2001). Introduction: The physician role in transition: is Hippocrates sick? Social Science and Medicine, 52, 171-173.

Gómez U., J. (2017). Prevalence of burnout syndrome in emergency nurses: a meta-analysis. Critical Care Nurse, 37(5), 1-9. doi: $10.4037 / \operatorname{ccn} 2017508$.

Grau. A., Suñer. R. y García, M.M. (2005). Desgaste profesional en personal sanitario y su relación con factores personales y ambientales. Gaceta Sanitaria, 19, 463-470.

Hernández, P. (2002). Estudio descriptivo del síndrome de burnout en personal de salud del Hospital Militar de Bogotá. Acta Colombiana de Psicología, 7, 71-83.

Hernández, R., Fernández, C. y Baptista, P. (2014). Metodología de la investigación. México: McGraw-Hill.

Kaschka, W., Korczak, D. y Broich, K. (2011). Mode diagnose burn-out. DeutschesÄrzteblatt, 108(46), 781-787. doi: 10.3238/ arztebl.2011.078.

Londoño, N., Rogers, H., Castilla, J., Posada, S., Ochoa, N., Jaramillo, M., Oliveros, M., Palacio, J., Palacio, J. y Aguirre A., D. (2012). Validación en Colombia del Cuestionario MOS de Apoyo Social. International Journal of Psychological Research, $5(1), 142-150$.

Maslach, C. (2009). Comprendiendo el burnout. Ciencia y Trabajo, 32, 37-44. 
Maslach, C. y Jackson, S. E. (1986). Maslach Burnout Inventory Manual (2nd ed.). Palo Alto, CA: Consulting Psychologists Press.

Matud, P. (2004). Gender differences in stress and coping styles. Personality \& Individual Differences, 37, 1401-1415.

Menezes, V.A., Fernández, B., Hernández, L., Ramos, F. y Contador, I. (2006). Resiliencia y el modelo burnout: engagement en cuidadores formales de ancianos. Psicothema, 18(4), 769-791.

Mingote, J., Moreno B. y Gálvez, M. (2004). Desgaste profesional y salud de los profesionales médicos: Revisión y propuestas de prevención. Medicina Clínica, 123, 265-270.

Moreno, B., González, J.L. y Garrosa, E. (2001). Desgaste profesional (burnout), personalidad y salud percibida. En J. Buendía y F. Ramos. (Eds.): Empleo, estrés y salud (pp. 1-35). Madrid: Psicología Pirámide.

Moreno J., B., González G., J.L. y Garrosa H., E. (2002). Variables sociodemográficas en el proceso de desgaste profesional de enfermería. Rol de Enfermería, 25(11), 18-26.

Muñoz, Y., Osorio, D., Robles, E. y Romero, Y. (2014). Sindrome de burnout en el personal de enfermería de un hospital de referencia Ibagué 2014. Tesis de pregrado Ibagué (Colombia): Universidad del Tolima.

Naranjo, M. (2009). Una revisión teórica sobre el estrés y algunos aspectos relevantes de éste en el ámbito educativo. Educación, 33(2), 171-190.

Notario, B., Martínez, V., Trillo, E., Pérez, M., Serrano, D. y García, J. (2014). Validity and reliability of the Spanish version of the 10-item CD-RISC in patients with fibromyalgia. Health and Quality of Life Outcomes, 1, 12-14. doi: 10.1186/1477$7525-12-14$.

Olmedo, E. (1997). Personalidad y afrontamiento. Determinantes del síndrome de estar quemado en profesionales de enfermería. Tesis doctoral inédita. La Laguna (España): Universidad de La Laguna.

Pérez N., M., Iruarrizaga, I. y Camuñas, N. (2001). Prevención y control del estrés en el cambio hospitalario (Conferencia 28). Memorias del I Congreso Virtual de Psiquiatría: Recuperado de http://www.psiquiatria.com/congreso/mesa/mesa28/conferencia /28_ci_c.htm.

Richardsen, A. y Burke, R. (1991) Occupational stress and job satisfaction among physicians: sex differences. Socscimed, 33(10), 1179-1187.

Ríos, M., Carrillo, C. y Sabuco, E. (2012). Resiliencia y síndrome de burnout en estudiantes de enfermería y su relación con variables sociodemográficas y de relación interpersonal. International Journal of Psychological Research, 5(1), 88-95.

Riveros, F., Bernal, L., Bohórquez, D., Vinaccia, S. y Quiceno, J. (2016). Validez de constructo y confiabilidad del Connor-Davidson Resilience Scale (Cd-Risc 10) en población colombiana con enfermedades crónicas. Salud y Sociedad, 7(2), 130-137.

Rodríguez, M., Rodríguez, R., Riveros, A., Rodríguez, M. y Pinzón, J. (2010). Síndrome de burnout y factores asociados en personal de salud en tres instituciones de Bogotá en diciembre de 2010. Tesis de pregrado en Medicina. Bogotá: Universidad del Rosario.

Rozo, M. (2007). Evaluación del síndrome de estrés asistencial en los profesionales de la salud en una institución hospitalaria de III nivel en la ciudad de Bogotá. Psychologia: Avances de la Disciplina, 1(1), 185-214.

Rueda P., C. y Vélez B., H. (2010). Características psicométricas de un cuestionario de estilo de afrontamiento. Típica, Boletín Electrónico de Salud Escolar, Artículo 5, 6(2).

Sabogal, K., Herrera, S. y Farfán, D. (2015). Estilo de vida y síndrome de burnout en trabajadores de la salud de una clínica privada de Lima. Revista Cientifica de Ciencias de la Salud, 8(1), 56-62.

Scheier, M. y Carver, C. (1985). Optimism, coping and health: Assessment and implications of generalized outcome expectancies. Health Psychology, 4, 219-247.

Siegrist, J. (1996). Adverse health effects of high-effort/low-reward conditions. Journal of Occupational Health Psychology, 1(1), 27-41.

Stewart A.L. y Ware, J.E. (Eds.). (1992). Measuring functioning and well-being: The Medical Outcomes Study Approach. Durham, N.C.: Duke University Press.

Taris, T., Peeters, M., Le Blanc, P., Schreurs, P. y Schaufeli, W. (2001). From inequity to burnout: the role of job stress. Journal of Occupational Health Psychology, 6(4), 303-323.

Vitaliano, P.P., Russo, J., Carr, J.E., Maiuro, R.D. y Becker, J. (1985). The ways of Coping Checklist: Revision and psychometric properties. Multivariate Behavioural Research, 20, 3-26.

Zenger, M., Finck, C., Zanon, C., Jimenez, W., Singer, S. y Hinz, A. (2013). Evaluationof the Latin American version of the Life Orientation Test-Revised. International Journal of Clinical and Health Psychology, 13, 243-252. 